\title{
Characterization of thermally induced transitions of collagen using second harmonic generation (SHG) microscopy
}

\author{
Yen Sun ${ }^{\mathrm{a}}$, Chih-Yuan Hsiao ${ }^{\mathrm{b}}$, Shu-Wen Teng ${ }^{\mathrm{a}}$, Wen Lo ${ }^{\mathrm{a}}$, Hsing-Yuan Tan ${ }^{\mathrm{c}}$, Sung-Jan Lin ${ }^{\mathrm{d}}$, Gwo-Jen \\ $\mathrm{Jan}^{\mathrm{b}}$, Wei-Chou Lin ${ }^{\mathrm{e}}$, Shiou-Hwa Jee ${ }^{\mathrm{d}, \mathrm{f}}$, Chen-Yuan Dong ${ }^{\mathrm{a}}$ \\ ${ }^{a}$ Department of Physics, National Taiwan University, Taipei 106, Taiwan \\ ${ }^{\mathrm{b}}$ Institute of Electro Optics, Department of Electrical Engineering, National Taiwan University, \\ Taipei 106, Taiwan \\ ${ }^{\mathrm{c}}$ Department of Ophthalmology, Chang Gung Memorial University, Linko 333, Taiwan \\ ${ }^{\mathrm{d}}$ Department of Dermatology, National Taiwan University, Taipei 100, Taiwan \\ ${ }^{\mathrm{e}}$ Department of Pathology, National Taiwan University Hospital, Taipei 100, Taiwan \\ ${ }^{\mathrm{f}}$ Department of Dermatology, College of Medicine, National Taiwan University, Taipei 100, Taiwan
}

\begin{abstract}
As a major component of the connective tissues, collagen fibers are responsible for various physiological functions inside the body. They provide support for the skin, partial focusing through the cornea, and coordinate movements via tendons, ligaments, and cartilages. In many medical procedures, thermal reorganization of the collagen structure is inevitable or desired. Therefore, the optimization of the therapeutic values of these procedures requires the characterization of thermal changes to collagen fibers. In this presentation, we use multiphoton microscopy to achieve this task. We will show that second harmonic generation (SHG) microscopy can characterize the thermally altered states of collagen and that they have potentials to be used in imaging applications in vivo.
\end{abstract}

Keywords: multiphoton microscopy, second-harmonic generation, collagen, thermal transition

To whom correspondence should be addressed: ${ }^{*}$ shiouhwa@ha.mc.ntu.edu.tw, *cydong@phys.ntu.edu.tw 


\section{INTRODUCTION}

\subsection{Multiphoton microscopy}

In the decade since the introduction of multiphoton microscopy, non-linear imaging modalities have gained tremendous popularity in the life sciences. A number of significant advantages are associated with this methodology. To begin with, the high incident photon flux necessary to induced non-linear optical effects from biological specimens limit sample excitation to the focal volume. By scanning the point-like excitation volume across specimens of interest, images with excellent axial depth discrimination can be achieved without using confocal apertures. Another advantage of the point-like excitation volume is the reduced overall sample photodamage. Another major advantage of multiphoton microscopy is due to the longer wavelength photons used for sample excitation. Unlike the ultraviolet or visible photons used in single-photon microscopy, the near-infrared photons commonly used in multiphoton imaging are scattered and absorbed less by tissue constituents. As a result, greater sample imaging depths can be achieved without invasive histological procedures. Finally, the wide spectral separation between the excitation and emission photons allows sample luminescence to be easily detected. ${ }^{1,2}$

In addition to multiphoton fluorescence excitation, another special class of important non-linear optical phenomenon has been explored for bioimaging applications. In general, the polarization effects associated with a material may be expressed as

$$
P_{i}=\chi_{i j}^{(1)} E_{j}+\chi_{i j k}^{(2)} E_{j} E_{k}+\chi_{i j k l}^{(3)} E_{j} E_{k} E_{l}+\ldots
$$

where $P_{i}$ and $E_{j}$ are the polarization and electric field components. $\chi_{i j}^{(1)}, \chi_{i j k}^{(2)}$, and $\chi_{i j k l}^{(3)}$ are the first, second, and third order susceptibility tensors, respectively. In second harmonic generation (SHG), structures lacking inversion symmetry have non-vanishing second order susceptibility tensor $\chi_{i j k}^{(2)}$. The polarization associated with this special class of materials convert incident photons into photons at half the wavelength. Biological structures such as collagen and muscle fibers have been shown to be effective generators of SHG signals. ${ }^{3,4}$

The unique advantages of non-linear microscopy have enabled this technique to be applied in a number of diverse fields in the life sciences including neurobiology, developmental biology, and deep tissue imaging. ${ }^{5,6,7}$

\subsection{Thermal effects on collagen integrity}

Among the different extracellular matrix (ECM) molecules, collagen is clearly one of the most important. Varying forms of collagen form an integral part of human physiology. Tendon, cornea, skin dermis, and cartilage are just a few examples where collagen fulfills structural and functional roles within the human physiology. A number of significant biomedical issues are associated with the damage and subsequent repair of collagen matrix. Specifically, in conductive keratoplasty, vision correction is achieved by heating of patient's cornea. Therefore, the development of an effective 
imaging technique to detect the structural changes to collagen matrix has significant values in biomedical applications. In the case of collagen, SHG imaging has a number of distinct advantages for the detection of its structural changes. First, the SHG signal is spectrally specific. For example, the generation of the SHG signal is exactly at half the wavelength of the incident excitation wavelength. If $780 \mathrm{~nm}$ light is used as the excitation source, the resulting SHG of the collagen matrix will be at $390 \mathrm{~nm}$. The spectral specific of SHG signal allows unequivocal identification of the SHG tissues of interests. In addition, since harmonic generation is a polarization effect not involving actual molecular transition, the sample photobleaching associated with relatively long lifetime of the excited states of molecules are eliminated. This feature is particularly attractive in the development of SHG microscopy into a minimally-invasive bioimaging technique.

Previously, a number of studies have used SHG intensity to characterizing the thermal damage to collagen and it was found that a structural transition occur at near $64^{\circ} \mathrm{C}$, where a decrease in SHG intensity seem to be associated with its denaturation. ${ }^{8,9}$ In this work, we hope that a combination of the images and measured SHG intensity can help us to clarify the detailed mechanisms when collagen matrix undergo thermal denaturation.

\section{MATAERIALS AND METHODS}

\subsection{Set-up of a multiphoton microscope}

The multiphoton microscopy used in this study is similar to a system described previously. ${ }^{10}$ In short, the $780 \mathrm{~nm}$ output of a diode-pumped (Millennia X, Spectra Physics, Mountain View, CA), titanium-sapphire (Tsunami, Spectra Physics) laser system is used as the excitation source. The laser light is guided toward a modified upright microscope system (E800, Nikon, Japan). Prior the entering the microscope, the angular deviation of the laser beam is varied by an X-y scanning system (Model 6220, Cambridge Technology, Cambridge, MA). The scanned beam is then beam expanded before being reflected by a dichroic mirror (700DCSPXRUV-3p, Chroma Technology, Brattleboro, VT) to over fill the back aperture of the focusing objective. In our study, a high numerical aperture, oil immersion objective (S Fluor 40x, NA 1.3, Nikon) was used to focus the laser beam for inducing the SHG signal. The generated SHG signal is collected in the back scattering geometry. To ensure equal SHG induction of the collagen fibers at different orientations, a quarter wave plate was used to covert the linearly polarized ti-sa laser into a circularly polarized beam. The average power of the laser beam is about $5.1 \mathrm{~mW}$ at the sample. The back-scattered SHG signal collected by the objective was further isolated using a combination of a short pass filter (E680SP, Chroma Technology) and a band pass filter (HQ390/20, Chroma Technology). The SHG signal was finally processed using a single-photon counting PMT (R7400P, Hamamatsu, Japan). To acquire large area multiphoton images of the thermally treated collagen, an x-y sample translation stage (H101, Prior Scientific, UK) was used to translate the specimen after each small area optical bean scan by the laser beam.

\subsection{Preparation of thermally treated collagen}

Rat tail tendon was used for this experiment. Small sections of the rat tail tendon was obtained and then treated in temperature-controlled PBS baths between 25 and $60{ }^{\circ} \mathrm{C}$ for 10 minutes. At the end of the thermal cycle, the rat tail tendon sections were removed and mounted using No. 1.5 cover glasses for viewing. To obtain a more thorough view of the degree of thermal damage, a 6 by 6 array of SHG images were acquired using the sample scanning stage and 
assembled. Representative images processed in this fashion are shown in Fig. 1. Also shown in the figure are the histological images. The average SHG intensity was calculated and plotted in Fig. 2.

\section{RESULTS AND DISCUSSIONS}

An examination of the SHG images in Fig. 1 shows a number of features characterizing thermal damage of collagen fibers. First, at the lower temperatures of 25 and $40{ }^{\circ} \mathrm{C}$, the collagen fibers seem not be affected the thermal treatment. Both the SHG and histological images show the well-organized striated patterns characteristic of the collagen fibers. However, at higher temperatures such as $52{ }^{\circ} \mathrm{C}$, both the SHG and histological images start to show local breakages of the striated collagen fibers (indicated by arrows). As the temperature increases, there tends to be an increase in the breakage of collagen fibers. At $57^{\circ} \mathrm{C}$, SHG image reveals that a multiple of local breakages have occurred along with small segments of second harmonic generating elements. At this temperature, the histological image reveals what appear to be two types of the collagen structural domains. There are regions where the fibrous structures remain intact (elliptical region) while other regions show a molten state of the collagen fiber without obvious striation patterns (rectangular region). At $60{ }^{\circ} \mathrm{C}$, almost all SHG elements have disappeared and the histological image reveals what appears to be a molten state of the rat tail tendon. These observations support the fact that at higher temperatures, domains within the rat tail tendon have undergone denaturation while other domains remain intact. Therefore, thermal denaturation within rat tail tendon does not occur sharply as one would expect from a phase transition. It is conceivable that different domains within the collagen posses different degree of temperature-dependent structural integrity.

In addition, the temperature-dependent SHG intensity plot (Fig. 2) suggests that the rat tail tendon collagen denaturation occurs sharply between 55 and $60{ }^{\circ} \mathrm{C}$. However, without the SHG images displayed in Fig. 2, the local heterogeneity of collagen thermal denaturation is difficult to reveal.

$25^{\circ} \mathrm{C}$
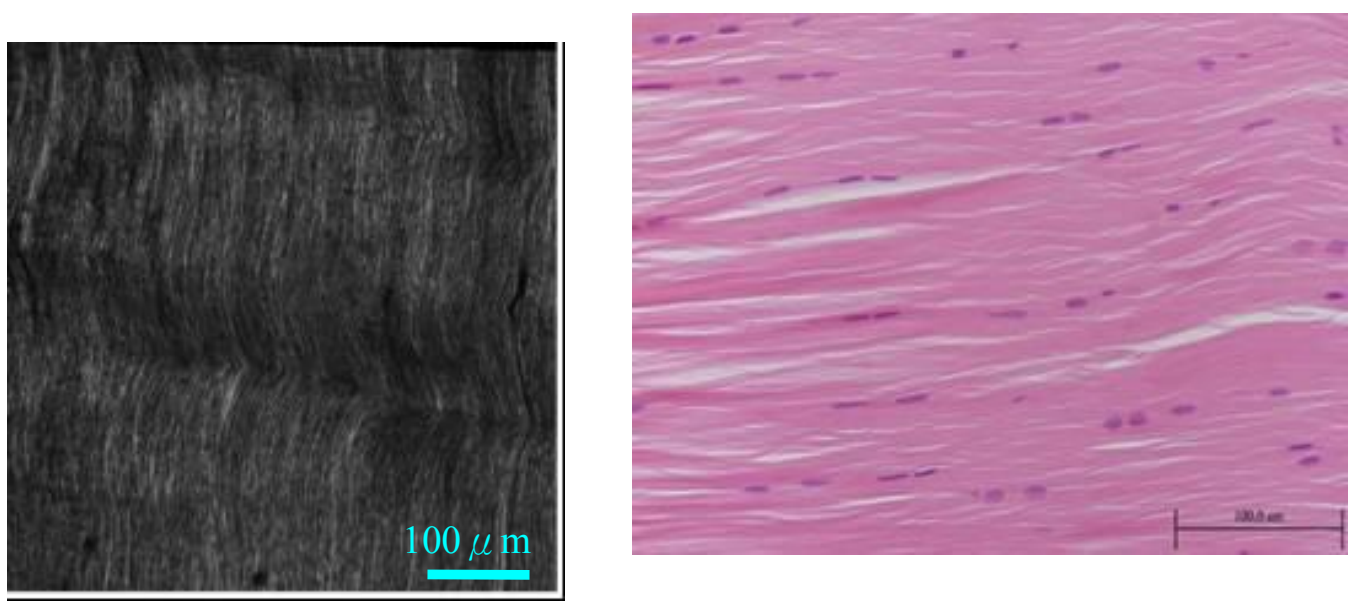


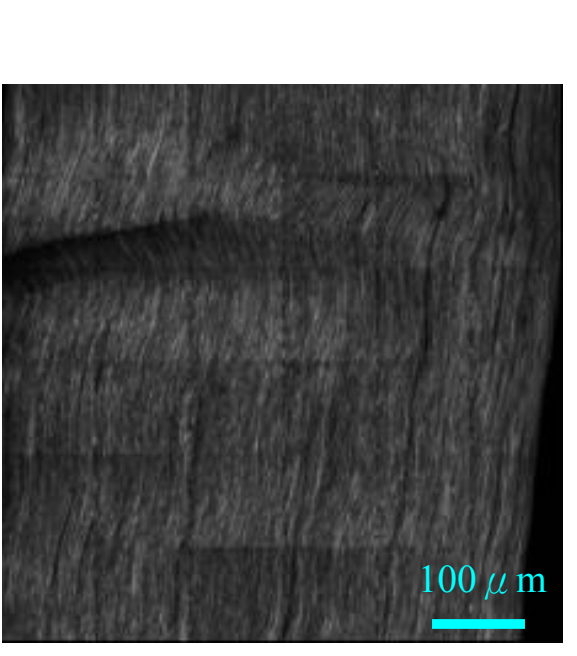

$40^{\circ} \mathrm{C}$

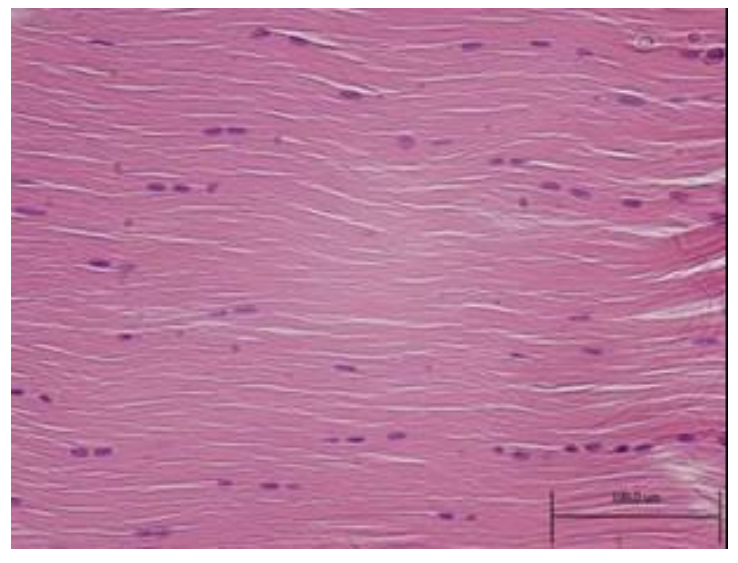

\section{$52^{\circ} \mathrm{C}$}
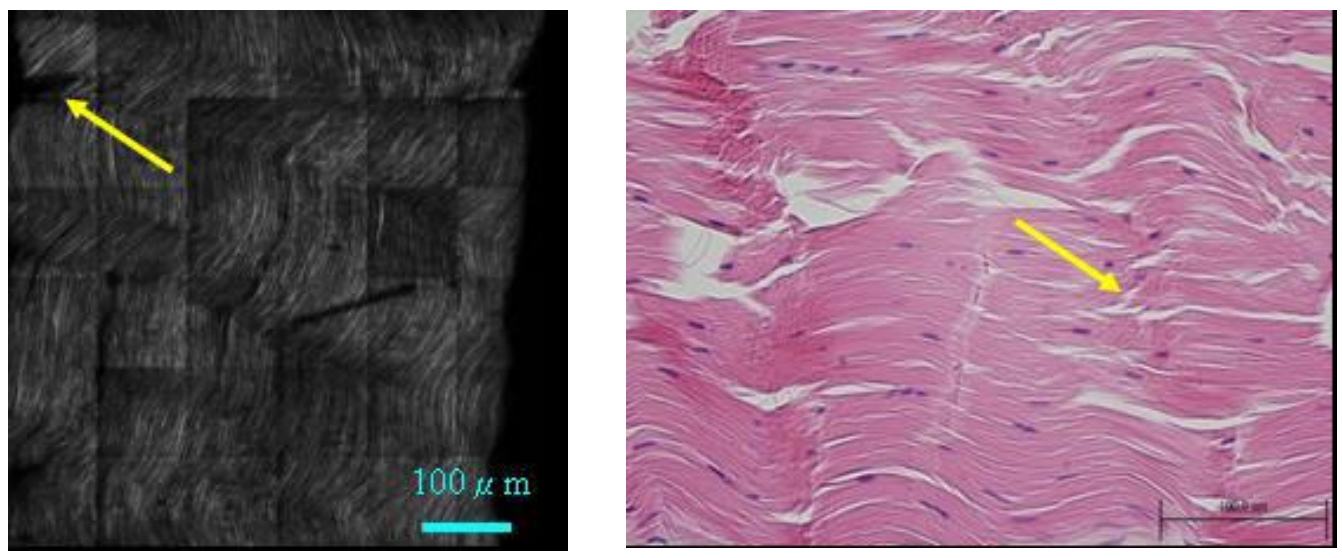

$55^{\circ} \mathrm{C}$
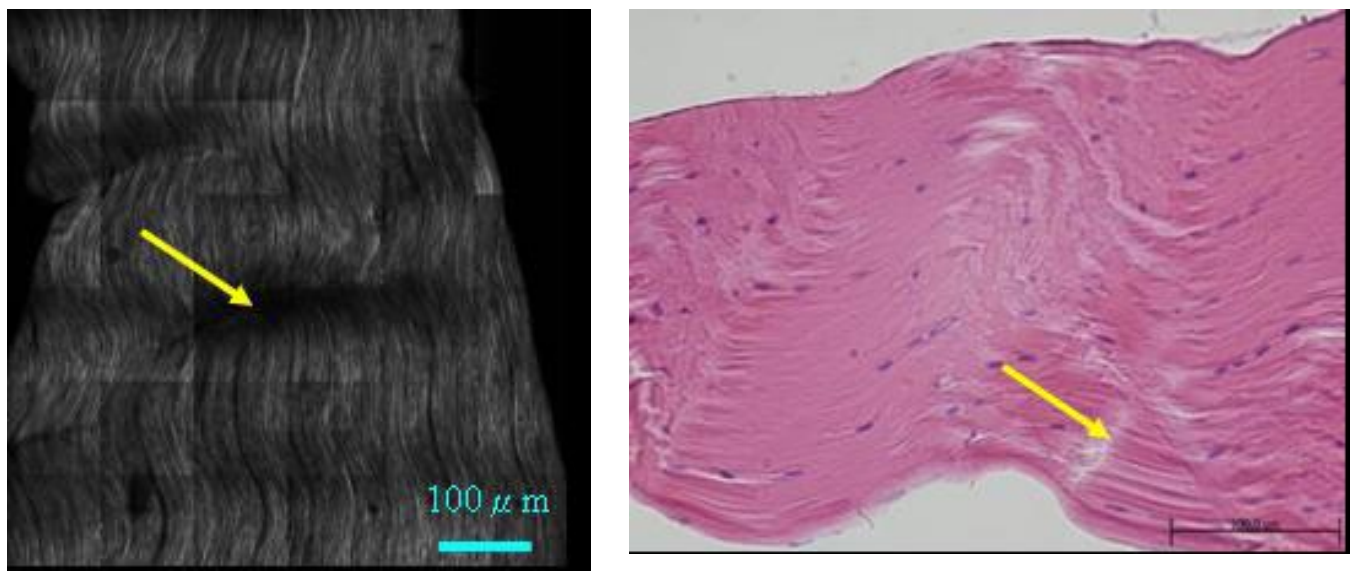

$57^{\circ} \mathrm{C}$ 

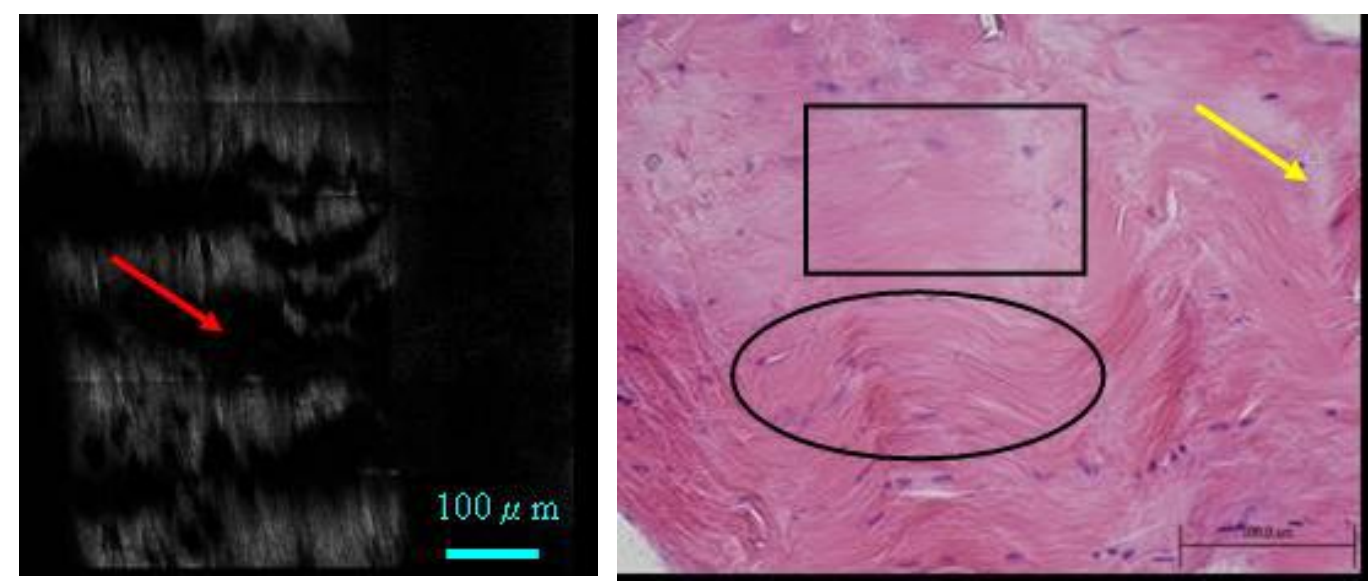

$60^{\circ} \mathrm{C}$
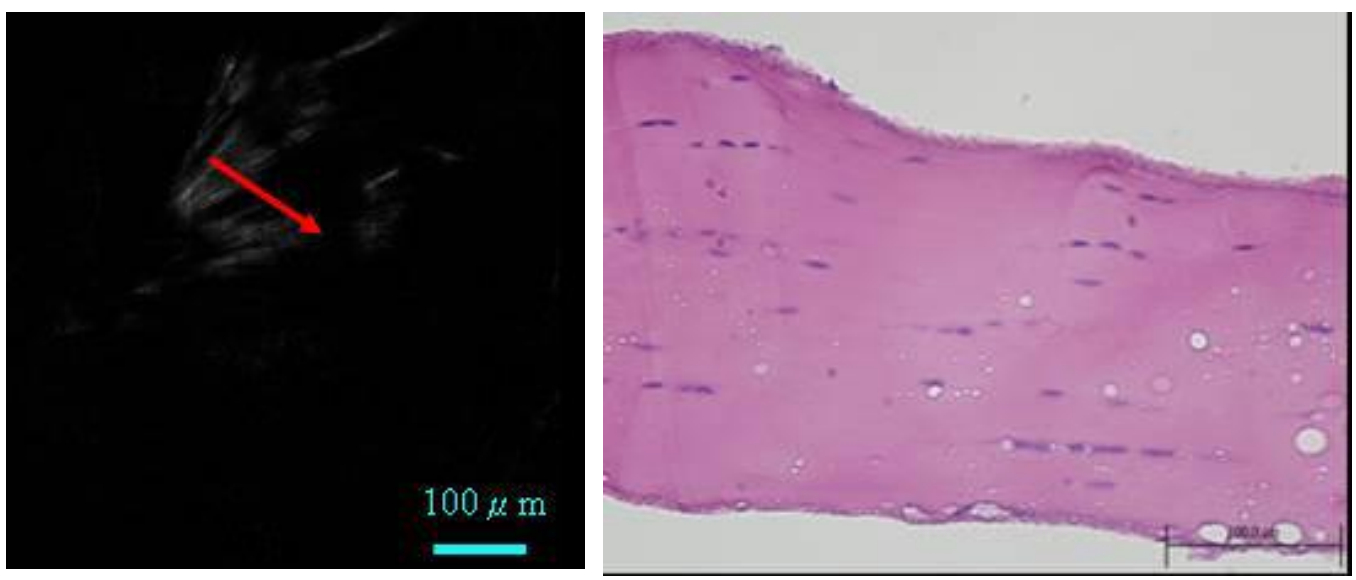

Fig. 1 Selected second harmonic generation (SHG) images (left column) and histological images (right column) of thermally treated rat tail tendon treated for 10 minutes in PBS buffer. 


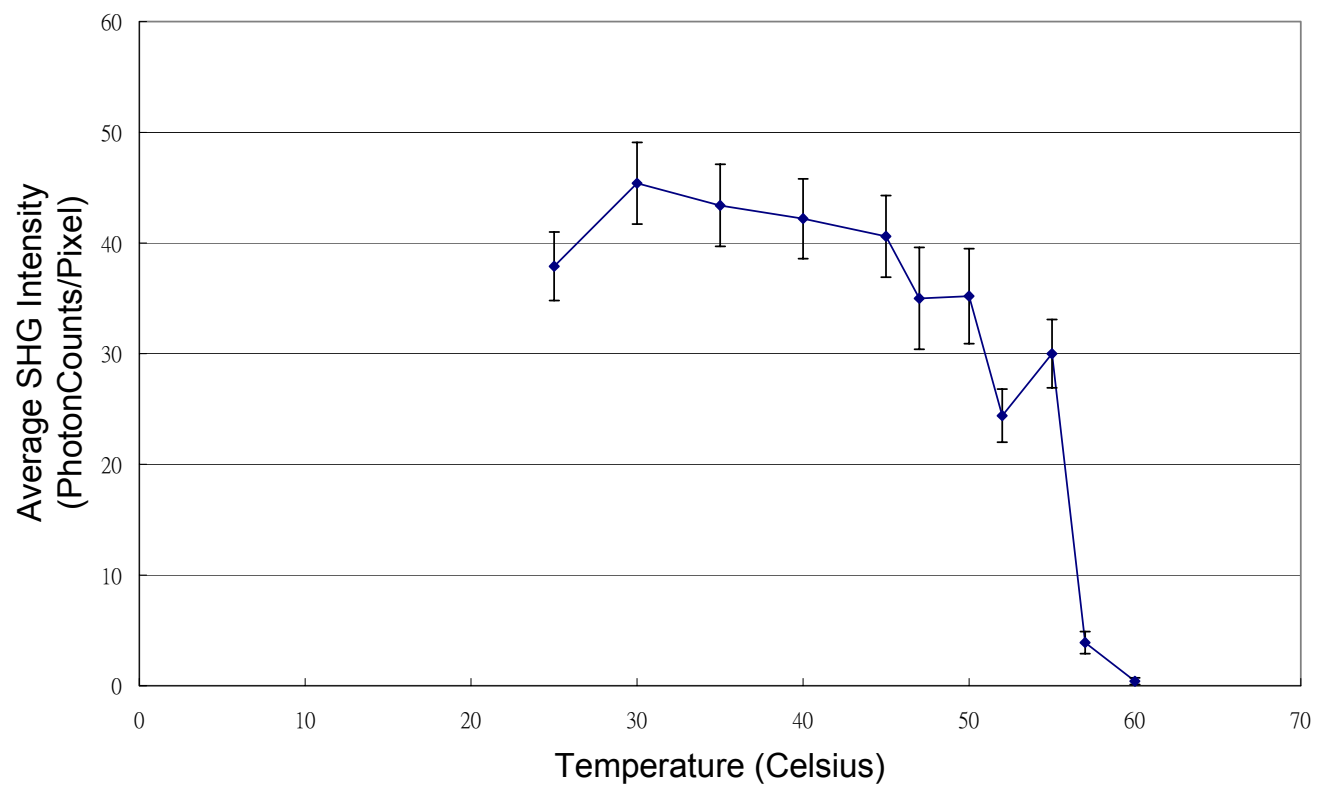

Fig. 2 Temperature dependence of rat tail tendon SHG intensity treated in PBS buffer for 10 minutes in the temperature range between 25 and $60^{\circ} \mathrm{C}$.

\section{CONCLUSIONS}

In conclusion, we have demonstrated that the non-linear imaging modality of second harmonic generation (SHG) microscopy is an effective technique for monitoring the thermally induced structural denaturation of collagen. Specifically, in the case of rat tail tendon, our results demonstrate that an increase in temperature lead to the local breakages of collagen fibers. With further increase in temperature, histological images show that the fibrous structures within different domains of the collagen become disrupted. These results seem to correlate to the decrease in SHG in the thermally treated specimens. The local variations of the thermal properties of collagen can not be revealed by the SHG intensity plot shown in Fig. 2. Our results suggest that the thermally induced structural denaturation of collagen occurs in a stepwise fashion, indicative of the fact that spatial heterogeneity in the thermal properties exists within collagen.

\section{Acknowledgment}

This work is supported by the National Research Program of Genomic Medicine of the National Science Council, Taiwan (NSC 92-2112-M-002-018 and NSC 92-3112-B-002-048).

\section{References}

1. W. Denk, J. H. Strickler, and W. W. Webb, “Two-photon laser scanning fluorescence microscopy,” Science. 248, pp. 73-76, 1990. 
2. P. T. C. So, C. Y. Dong, CY, B. R. Masters, and K. M. Berland, "Two-photon excitation fluorescence microscopy," in Annual Review of Biomedical Engineering, volume 2, pp. 399-429. Annual Reviews, Palo, Alto, 2000.

3. P. J. Campagnola and L. M. Loew, Nature Biotechnology 21(11), pp. 1356-1360 (2003).

4. A. Zoumi, A. Yeh, and B. J. Tromberg, Proceedings of the National Academy of Sciences of the United States of America 99(17), pp. 11014-11019 (2002).

5. J. M. Squirrell, D. L. Wokosin, J. G. White, and B. D. Bavister, "Long-term two-photon fluorescence imaging of mammalian embryos without compromising viability," Nat. Biotech. 17, pp. 763-767, 1999.

6. K. Svoboda, K, W. Denk, D. Kleinfeld, and D. W. Tank, "In vivo dentritic calcium dynamics in neocortical pyramidal neurons," Nature. 385, pp. 161-165, 1997.

7. P. T. C. So, H. Kim, and I. E. Kochevar, "Two-photon deep tissue ex vivo imaging of mouse dermal and subcutaneous structures," Opt. Exp. 3, 339-350, 1998.

8. T. Theodossiou, G. S. Rapti, V. Hovhannisyan, E. Georgiou, K. Politopoulos, and D. Yova, Lasers in Medical Science 17(1), pp. 34-41 (2002).

9. B. M. Kim, J. Eichler, K. M. Reiser, A. M. Rubenchik, and L. B. Da Silva, Lasers in Surgery and Medicine 27(4), pp. 329-335 (2000).

10. Y. Sun, J. W. Su, W. Lo, S. J. Lin, S. H. Jee, and C. Y. Dong, Optics Express, 11(25), pp. 3377- (2003). 